\title{
A NEW ALGORITHM FOR LONG BASELINE KINEMATIC POSITIONING WITH DUAL FREQUENCY GPS/GNSS RECEIVERS
}

\author{
Hiroshi Isshiki \\ IMA (Institute of Mathematical Analysis) \\ B507, 3-11, Nishiyamadai 6-Chome, Osaka-Sayama, \\ Osaka 589-0022, Japan \\ Tel \& Fax +81-72-367-7139 \\ Email: isshiki@dab.hi-ho.ne.jp \\ Jinling Wang \\ School of Surveying \& Spatial Information Systems \\ The University of New South Wales \\ Sydney, NSW 2052, Australia \\ Tel: $\quad 61-2-93854203$ \\ Fax: 61293137493 \\ Email: Jinling.Wang@unsw.edu.au
}

\begin{abstract}
Smoothing pseudo ranges with ionosphere-free combinations of phase ranges can be useful for long rang positioning, since this new smoothing process can significantly remove the effect of ionosphere delays in positioning applications. The smoothing process can be conducted without being affected by cycle slips, though it uses phase ranges. Therefore, it can provide a robust precise positioning solution which is not affected by cycle slips. The multipath effects may also be reduced, if the averaging interval taken is long enough. If the low frequency noise components and the hardware biases in pseudo ranges are reduced, the positioning performance with the smoothed pseudo ranges may be promising. In future GNSS receivers, both the noises and the hardware biases in pseudo ranges will be significantly reduced. Then, the positioning based on the proposed algorithm will be very useful.
\end{abstract}

\section{INTRODUCTION}

Generally speaking, smoothing pseudo ranges by phase ranges will be impacted by ionosphere delays. However, if we smooth pseudo ranges by ionosphere free combinations of phase ranges, the smoothing is not affected by ionosphere delays. So, it may eliminate the systematic errors in the pseudo ranges significantly. This feature can be utilized for long baseline kinematic positioning.

Since the phase ranges are used in the form of the differences of successive observables, the cycle slips may be detected and thus, their effects may be eliminated during the calculations. So, we can make a robust algorithm which is not affected by cycle slips. Furthermore, the multipath effects may also be reduced, if the averaging interval is taken long enough. 
However, the hardware biases in the pseudo ranges may affect the positioning result. The canceling or elimination of such biases by hardware or by software is important. In future GNSS receivers, the noises and the biases will become much smaller, and therefore, positioning with the pseudo ranges smoothed by the ionosphere free combination of the phase ranges can be promising.

\section{THEORY}

In the following discussions, the ionosphere free combinations of the pseudo ranges are used. This may be useful in long baseline positioning. However, in the case of the short baseline positioning, $C / A$ (or $P_{1}$ below) code itself would be more convenient, since the ionosphere delays may be small in this case, if the differential positioning is used.

Ionosphere free combinations of pseudo range $P_{I \alpha \beta}^{i j}$ and phase range $\Phi_{I \alpha \beta}^{i j}$ double differenced between satellites $i$ and $j$ and receivers $\alpha$ and $\beta$ are given as

$$
\begin{gathered}
P_{I \alpha \beta}^{i j} \equiv \frac{f_{1}^{2} P_{1 \alpha \beta}^{i j}-f_{2}^{2} P_{2 \alpha \beta}^{i j}}{f_{1}{ }^{i j}-f_{2}{ }^{2}}=\rho_{\alpha \beta}^{i j}+T_{\alpha \beta}^{i j}+e_{I \alpha \beta}^{i j}, \\
\Phi_{I \alpha \beta}^{i j} \equiv \frac{f_{1}^{2} \Phi_{1 \alpha \beta}^{i j}-f_{2}{ }^{2} \Phi_{2 \alpha \beta}^{i j}}{f_{1}^{2}-f_{2}{ }^{2}}=\rho_{\alpha \beta}^{i j}+T_{\alpha \beta}^{i j}+\frac{c\left(f_{1} N_{1 \alpha \beta}^{i j}-f_{2} N_{2 \alpha \beta}^{i j}\right)}{f_{1}^{2}-f_{2}{ }^{i j}}+\varepsilon_{I \alpha \beta}^{i j},
\end{gathered}
$$

Where

$P_{1 \alpha}^{i}$ and $P_{2 \alpha}^{i}$ : Pseudo ranges in $L_{1}$ and $L_{2}$ waves between the satellite $i$ and the receiver $\alpha$. In the present GPS, $P_{1}$ and $P_{2}$ correspond to $C / A$ and $P_{2}$ code ranges respectively,

$\Phi_{1 \alpha}^{i}$ and $\Phi_{2 \alpha}^{i}$ : Phase ranges in $L_{1}$ and $L_{2}$ waves between the satellite $i$ and the receiver $\alpha$,

$f_{1}$ and $f_{2}:$ Frequencies of $L_{1}$ and $L_{2}$ waves,

$\rho_{\alpha}^{i}$ : Distance between the satellite $i$ and the receiver $\alpha$,

$T_{\alpha}^{i}$ : Troposphere delay between the satellite $i$ and the receiver $\alpha$,

$N_{\alpha}^{i}$ : Ambiguity of the phase range between the satellite $i$ and the receiver $\alpha$,

$e_{I \alpha}^{i}$ : Error in the pseudo range $P_{I \alpha}^{i}$,

$\varepsilon_{I \alpha}^{i}$ : Error in the phase range $\Phi_{I \alpha}^{i}$.

We apply a smoothing or low pass filter (Misra and Enge, 2001):

$$
\begin{aligned}
\bar{P}_{I \alpha \beta}^{i j}(t)= & \frac{1}{M} P_{I \alpha \beta}^{i j}(t)+\frac{M-1}{M}\left[\bar{P}_{I \alpha \beta}^{i j}(t-1)+\left(\Phi_{I \alpha \beta}^{i j}(t)-\Phi_{I \alpha \beta}^{i j}(t-1)\right)\right], \\
& \bar{P}_{I \alpha \beta}^{i j}(0)=P_{I \alpha \beta}^{i j}(0),
\end{aligned}
$$

where $\bar{P}_{I \alpha \beta}^{i j}(t)$ is the smoothed quantity of $P_{I \alpha \beta}^{i j}(t)$, and $M$ is a parameter determining the weights of the pseudo and phase ranges, since

$$
\bar{P}_{I \alpha \beta}^{i j}(t)-\bar{P}_{I \alpha \beta}^{i j}(t-1)=\frac{1}{M}\left(P_{I \alpha \beta}^{i j}(t)-\bar{P}_{I \alpha \beta}^{i j}(t-1)\right)+\frac{M-1}{M}\left(\Phi_{I \alpha \beta}^{i j}(t)-\Phi_{I \alpha \beta}^{i j}(t-1)\right) .
$$


When $M$ is the bigger, the weight of the phase ranges is the bigger and the variation in $\bar{P}_{I \alpha \beta}^{i j}(t)$ is reduced. The random errors in the pseudo ranges are reduced drastically as shown in numerical examples in the next section.

Since the above mentioned filter is a low pass filter, low frequency components in the noises $e_{I \alpha \beta}^{i j}$ and $\varepsilon_{I \alpha \beta}^{i j}$ are unfavorable. We need a big value of $M$ to reduce these components. The hardware biases or direct current components of noises in the pseudo ranges cannot be reduced by the above mentioned filter. The magnitude of noise $e_{I \alpha \beta}^{i j}$ is about three times bigger than that of $e_{1 \alpha \beta}^{i j}$, if the magnitude of $e_{1 \alpha \beta}^{i j}$ is equal to that of $e_{2 \alpha \beta}^{i j}$. So, in the case of a short baseline positioning, $\bar{P}_{1 \alpha \beta}^{i j}(t)$ may be more useful than $\bar{P}_{I \alpha \beta}^{i j}(t)$.

The baseline lengths, $\operatorname{dr}(\mathrm{CI}), \operatorname{dr}(\mathrm{C} 1)$ and $\operatorname{dr}(\mathrm{P} 2)$, obtained by using $P_{I \alpha \beta}^{i j}, P_{1 \alpha \beta}^{i j}$ and $P_{2 \alpha \beta}^{i j}$ are shown in Figure 1. As can be seen in this figure, $P_{2 \alpha \beta}^{i j}$ seems to be rather noisier than we expect for the present GPS where $L_{2}$ wave is not allowed for civil use originally. In future GNSS, the noises in pseudo ranges will be significantly reduced.

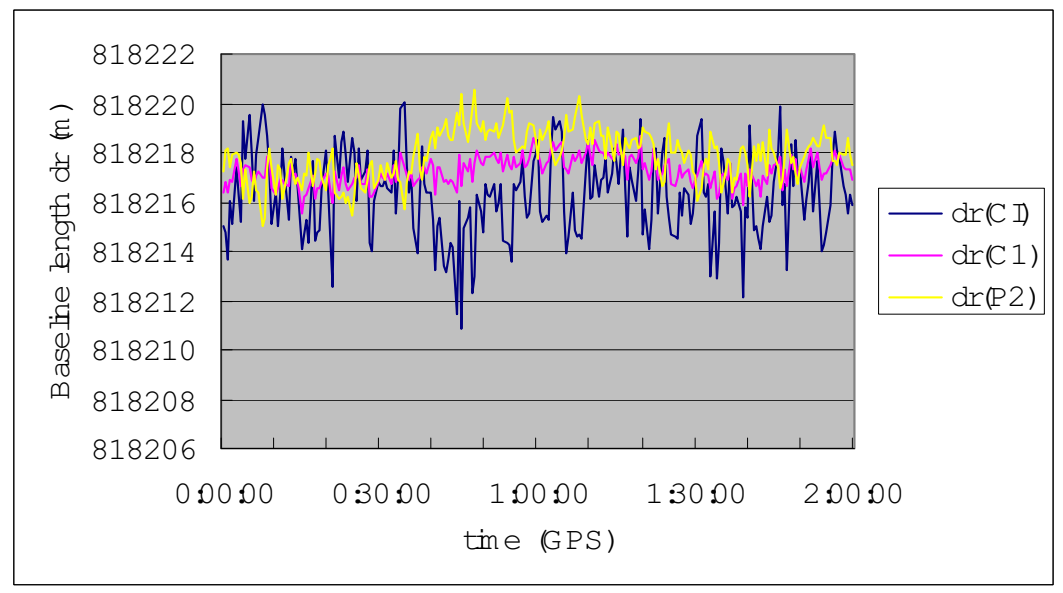

Fig. 1. Baseline lengths $d r$ obtained by $P_{I \alpha \beta}^{i j}, P_{1 \alpha \beta}^{i j}$ and $P_{2 \alpha \beta}^{i j}$.

(The data were obtained by GEONET. Sapporo and Ichikawa, 2002.12.06, Epoch=30sec).

\section{NUMERICAL EXAMPLES}

\subsection{RESULTS FOR JAPANESE DATA}

The data were obtained by GEONET (http://terras.gsi.go.jp/ja/index.html). The stations are Sapporo (Sppr) and Ichikawa (Ichk), and the baseline length is $818216.61 \mathrm{~m}$. The date and time of the observation are 2002.12.06 and 00:00:00-02:05:00 (GPS time), and the epoch is 30sec. Data for satellites 5, 6, 14, 25 and 30 were used. $M$ in equation (3) is assumed to be 30,60 and 120 epochs. The troposphere delays were corrected by Saastamoinen model with the standard condition, and the precise orbits were used. The effects of the troposphere delays and the accuracies of the orbits are to be briefly discussed in section 3.3 below. The effects of the troposphere delays are rather big, but the accuracy of the orbits is not so important since the precision of the positioning is at the decimeter level. 
The effects of smoothing are shown in Figures 2, 3, and 4. $P_{I \alpha \beta}^{i j}(t)-\Phi_{I \alpha \beta}^{i j}(t)$ as blue lines, and $\bar{P}_{I \alpha \beta}^{i j}(t)-\Phi_{I \alpha \beta}^{i j}(t)$ as magenta lines are shown in these figures, respectively. For $\Phi_{I \alpha \beta}^{i j}(t)$, the correct ambiguities obtained in the static positioning solutions of $\Phi_{I \alpha \beta}^{i j}(t)$ are used. As $M$ becomes large, $\bar{P}_{I \alpha \beta}^{i j}(t)-\Phi_{I \alpha \beta}^{i j}(t)$ becomes more slowly varying (to appear to be constant). This corresponds to the ambiguity in the phase range.

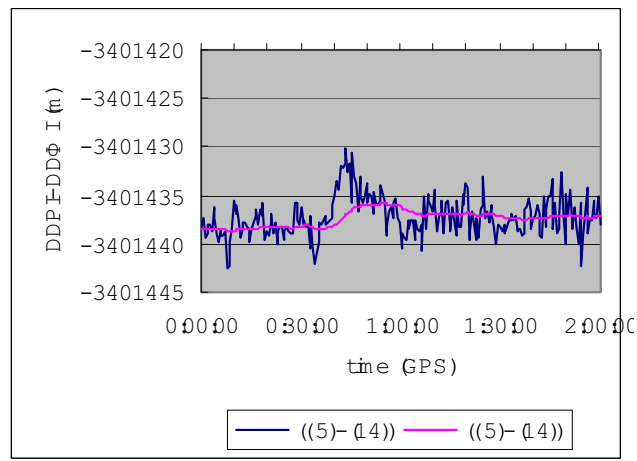

(a)Satellites (or SV, or PRN) 5 and 14

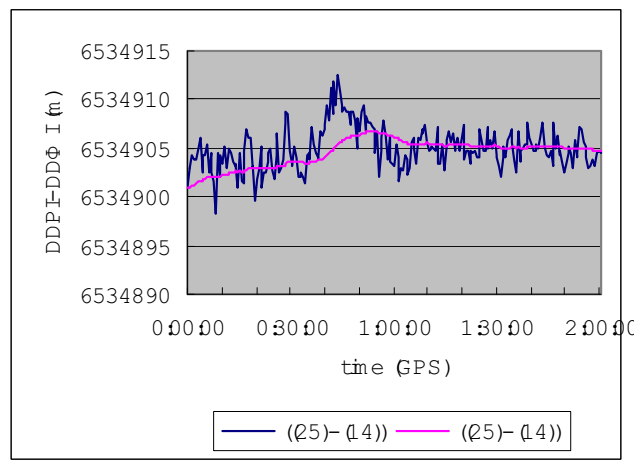

(c) Satellites 25 and 14

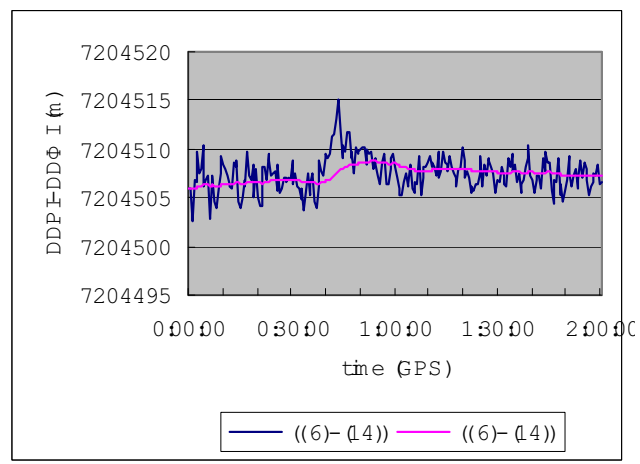

(b) Satellites 6 and 14

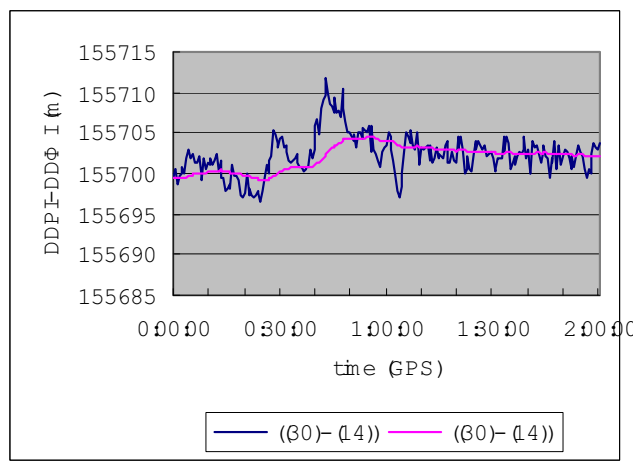

(d) Satellites 30 and 14

Fig. 2. $P_{I \alpha \beta}^{i j}(t)-\Phi_{I \alpha \beta}^{i j}(t)$ (blue) and $\bar{P}_{I \alpha \beta}^{i j}(t)-\Phi_{I \alpha \beta}^{i j}(t)$ (magenta) when $M=30$. 


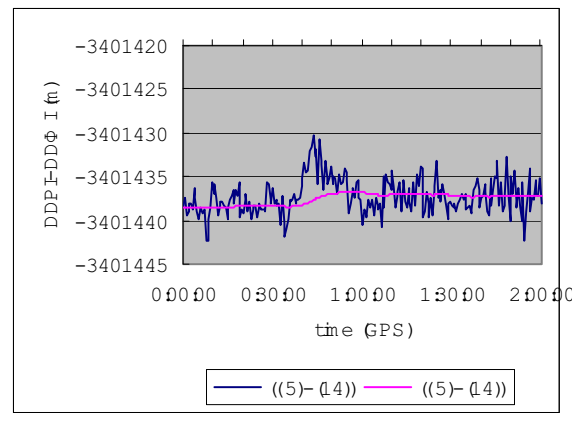

(a) Satellites 5 and 14

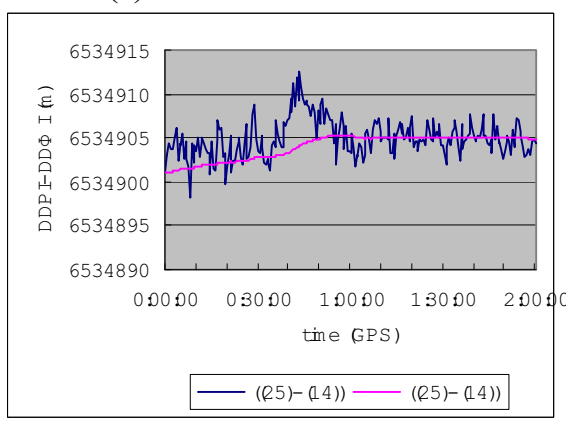

(c) Satellites 25 and 14

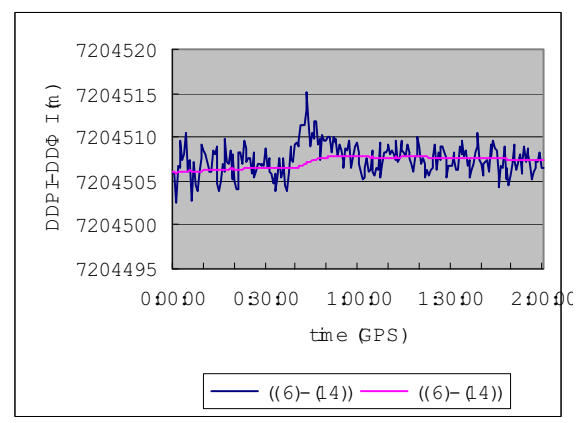

(b) Satellites 6 and 14

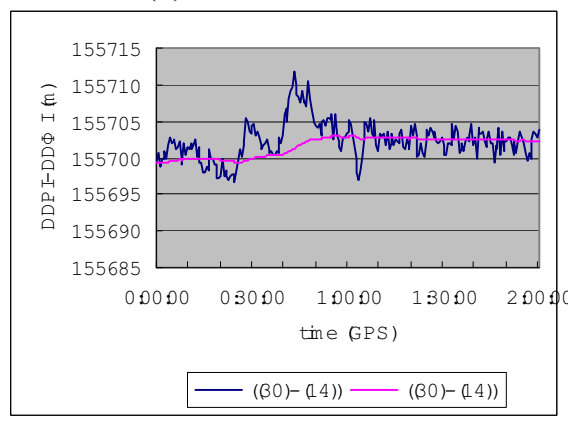

(d) Satellites 30 and 14

Fig. 3. $P_{I \alpha \beta}^{i j}(t)-\Phi_{I \alpha \beta}^{i j}(t)$ (blue) and $\bar{P}_{I \alpha \beta}^{i j}(t)-\Phi_{I \alpha \beta}^{i j}(t)$ (magenta) when $M=60$.

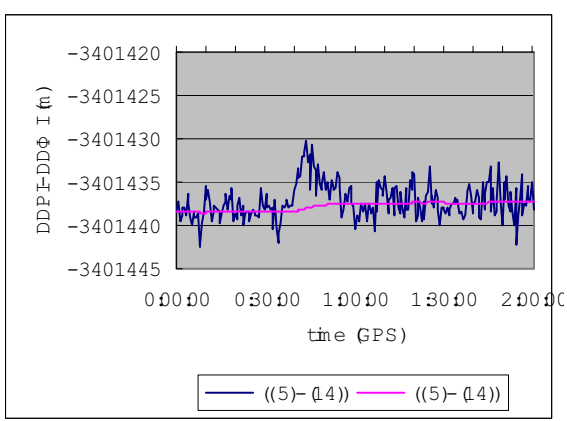

(a) Satellites 5 and 14

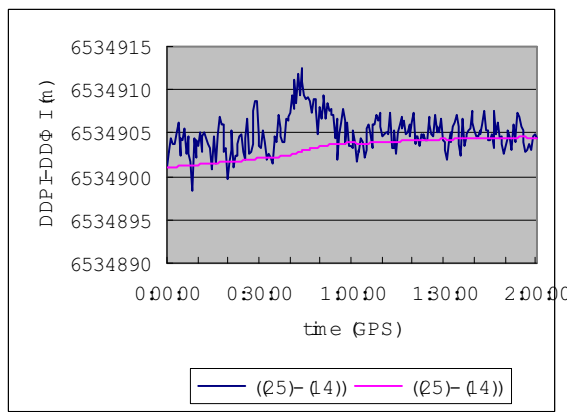

(c) Satellites 25 and 14

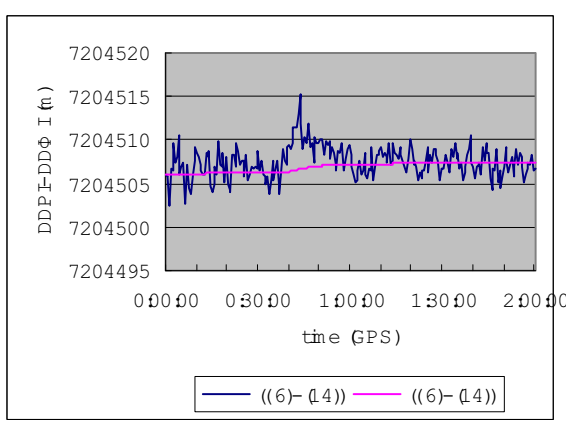

(b) Satellites 6 and 14

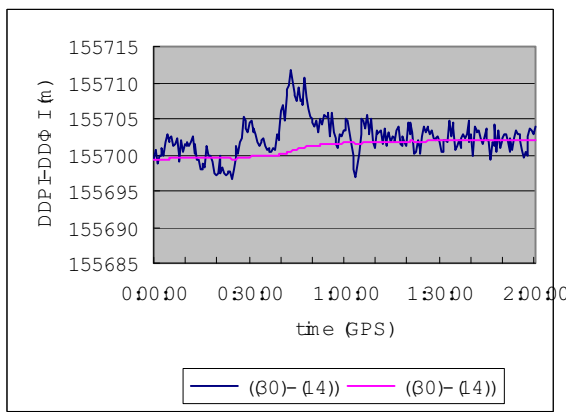

(d) Satellites 30 and 14

Fig. 4. $P_{I \alpha \beta}^{i j}(t)-\Phi_{I \alpha \beta}^{i j}(t)$ (blue) and $\bar{P}_{I \alpha \beta}^{i j}(t)-\Phi_{I \alpha \beta}^{i j}(t)$ (magenta) when $M=120$. 
In Figure 5, the positioning results are shown. For $\Phi_{I \alpha \beta}{ }^{i j}(t)$ and $\Phi_{1 \alpha \beta}{ }^{i j}(t)$, the correct ambiguities obtained by the static positioning of $\Phi_{I \alpha \beta}^{i j}(t)$ are used. The high frequency noise components in the positioning results by $\bar{P}_{I \alpha \beta}^{i j}$ are reduced reasonably, and the accuracy of the positioning results by $\bar{P}_{I \alpha \beta}^{i j}$ is better than that by $P_{1 \alpha \beta}^{i j}$. However, a rather big value for $M$ is required to suppress the low frequency components. In future GNSS system, the situation would be improved. A summary of the positioning results is given in Table 1 .

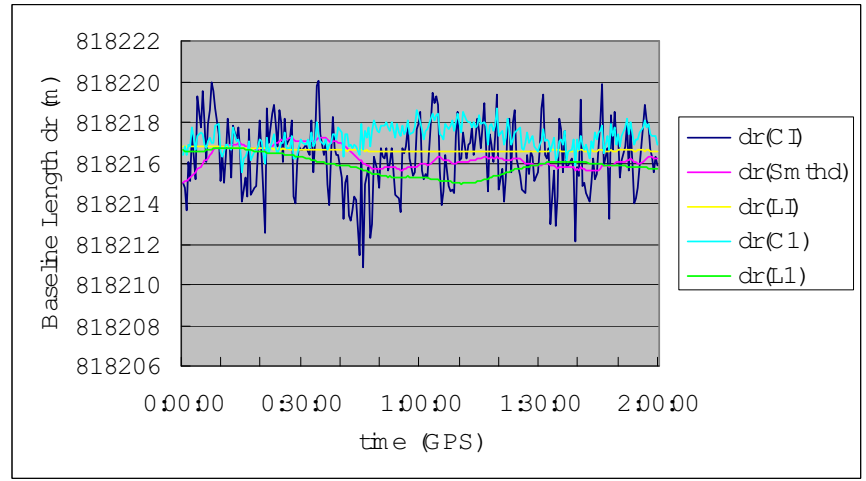

(a) $\mathrm{M}=30$

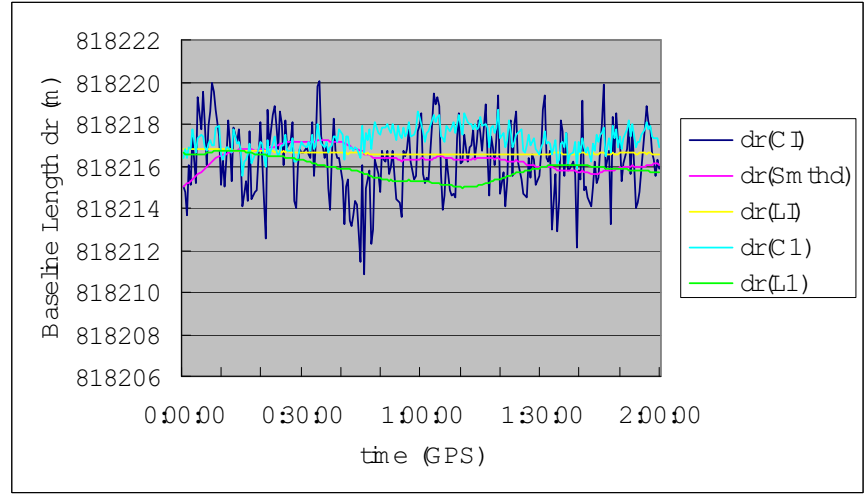

(b) $M=60$

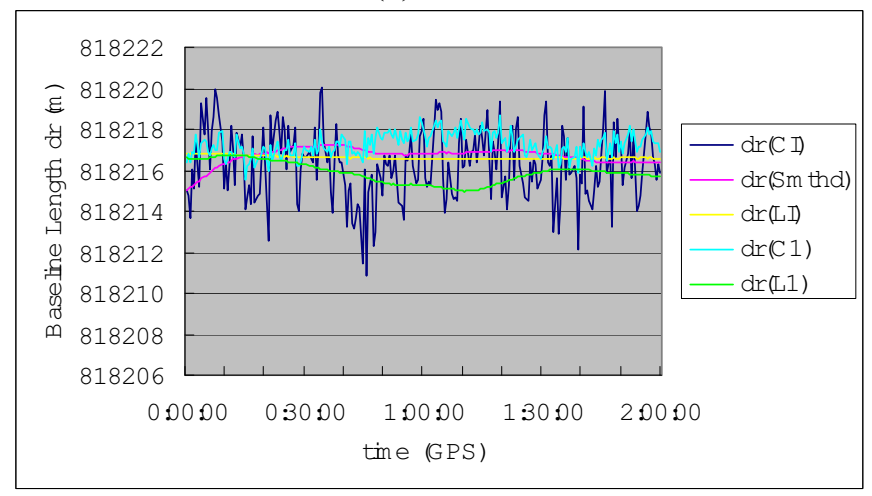

(c) $\mathrm{M}=120$

Fig. 5. Baseline lengths $d r$ obtained by $P_{I \alpha \beta}^{i j}, \bar{P}_{I \alpha \beta}^{i j}, \Phi_{I \alpha \beta}^{i j}, P_{1 \alpha \beta}^{i j}$ and $\Phi_{1 \alpha \beta}^{i j}$. 
Table 1a. Summary of the positioning results (2002.12.06, SpprIchk).

\begin{tabular}{|c|c|c|c|c|c|}
\hline \multirow[b]{2}{*}{ Baseline } & \multirow{2}{*}{$\begin{array}{l}\text { Averaging } \\
\text { interval }\end{array}$} & $\mathrm{C} 1$ & $\mathrm{CI}$ & CI-Smthd & LI \\
\hline & & $\begin{array}{c}\text { Mean } \\
\text { (Std. dev.) }\end{array}$ & $\begin{array}{c}\text { Mean } \\
\text { (Std. dev.) }\end{array}$ & $\begin{array}{c}\text { Mean } \\
\text { (Std. dev.) }\end{array}$ & Static \\
\hline $\mathrm{M}=30$ & $\begin{array}{l}0: 15: 00- \\
2: 00: 00\end{array}$ & $\begin{array}{c}818217.29 \\
(0.58)\end{array}$ & $\begin{array}{c}818216.18 \\
(1.68)\end{array}$ & $\begin{array}{c}818216.23 \\
(0.48)\end{array}$ & 818216.60 \\
\hline$M=60$ & $\begin{array}{l}0: 30: 00- \\
2: 00: 00\end{array}$ & $\begin{array}{c}818217.39 \\
(0.57)\end{array}$ & $\begin{array}{c}818216.15 \\
(1.69)\end{array}$ & $\begin{array}{c}818216.29 \\
(0.49)\end{array}$ & ditto \\
\hline $\mathrm{M}=120$ & $\begin{array}{l}1: 00: 00- \\
2: 00: 00\end{array}$ & $\begin{array}{c}818217.40 \\
(0.56)\end{array}$ & $\begin{array}{c}818216.36 \\
(1.58)\end{array}$ & $\begin{array}{c}818216.69 \\
(0.22)\end{array}$ & ditto \\
\hline
\end{tabular}

Table 1b. Difference of mean baseline length from the static results of LI

(2002.12.06, 0:00:00-2:00:00)

\begin{tabular}{|c|c|c|c|c|}
\hline Baseline & $\begin{array}{c}\text { Averaging } \\
\text { interval }\end{array}$ & $\begin{array}{c}\mathrm{C} 1 \\
(\mathrm{~m})\end{array}$ & $\begin{array}{c}\mathrm{CI} \\
(\mathrm{m})\end{array}$ & $\begin{array}{c}\text { CI-Smthd } \\
(\mathrm{m})\end{array}$ \\
\hline $\mathrm{M}=30$ & $0: 15: 00-2: 00: 00$ & +0.69 & -0.42 & -0.37 \\
\hline $\mathrm{M}=60$ & $0: 30: 00-2: 00: 00$ & +0.79 & -0.45 & -0.31 \\
\hline $\mathrm{M}=120$ & $1: 00: 00-2: 00: 00$ & +0.80 & -0.24 & +0.09 \\
\hline
\end{tabular}

Table 1c. Standard deviation (2002.12.06, 0:00:00-2:00:00)

\begin{tabular}{|c|c|c|c|c|}
\hline Baseline & $\begin{array}{c}\text { Averaging } \\
\text { interval }\end{array}$ & $\begin{array}{c}\mathrm{C} 1 \\
(\mathrm{~m})\end{array}$ & $\begin{array}{c}\mathrm{CI} \\
(\mathrm{m})\end{array}$ & $\begin{array}{c}\text { CI-Smthd } \\
(\mathrm{m})\end{array}$ \\
\hline $\mathrm{M}=30$ & $0: 15: 00-2: 00: 00$ & 0.58 & 1.68 & 0.48 \\
\hline $\mathrm{M}=60$ & $0: 30: 00-2: 00: 00$ & 0.57 & 1.69 & 0.49 \\
\hline $\mathrm{M}=120$ & $1: 00: 00-2: 00: 00$ & 0.56 & 1.58 & 0.22 \\
\hline
\end{tabular}

Similar calculations were conducted for the data obtained by GEONET on Nov. 6, 2006. The stations are Sapporo (Sppr) and Ichikawa (Ichk), and the observation time is 0:00:00- 2:00:00, and the data for satellites 1, 6, 7, 16 and 31 were used. The ionosphere was active on Dec. 6, 2002, but rather quiet on Nov 6, 2006. So, the effects of the ionosphere delay on the positioning are rather small as shown in Figure 6 and Table 2. The results by the present method are better than those obtained by using C1-ranges, but the differences are small. 


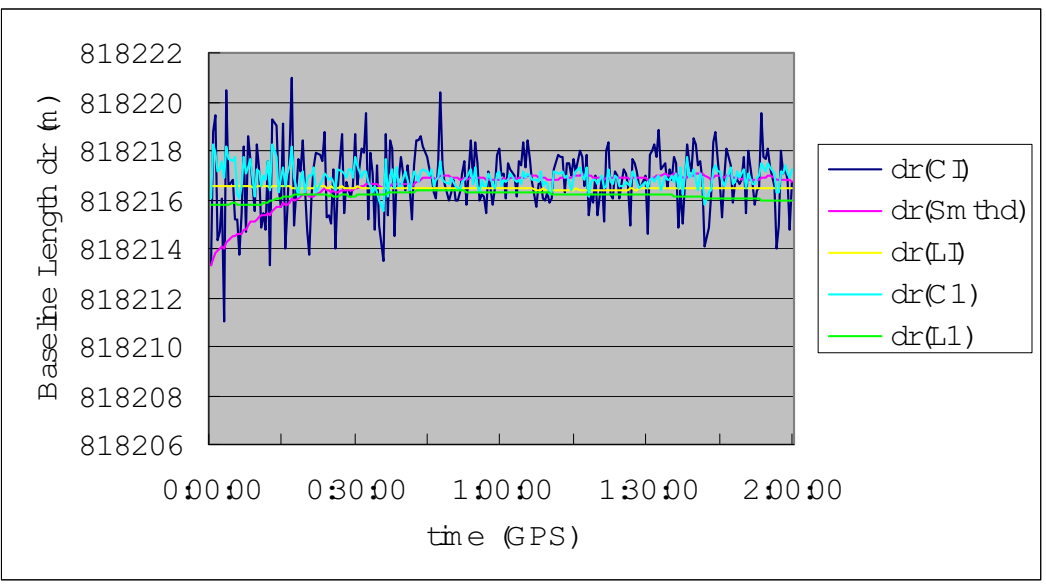

(a) $\mathrm{M}=30$

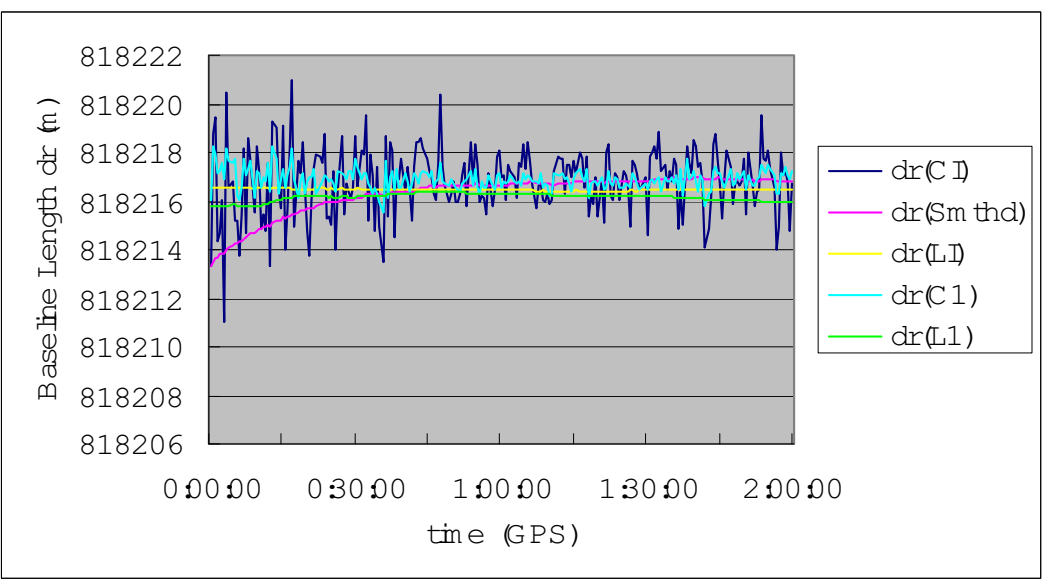

(b) $M=60$

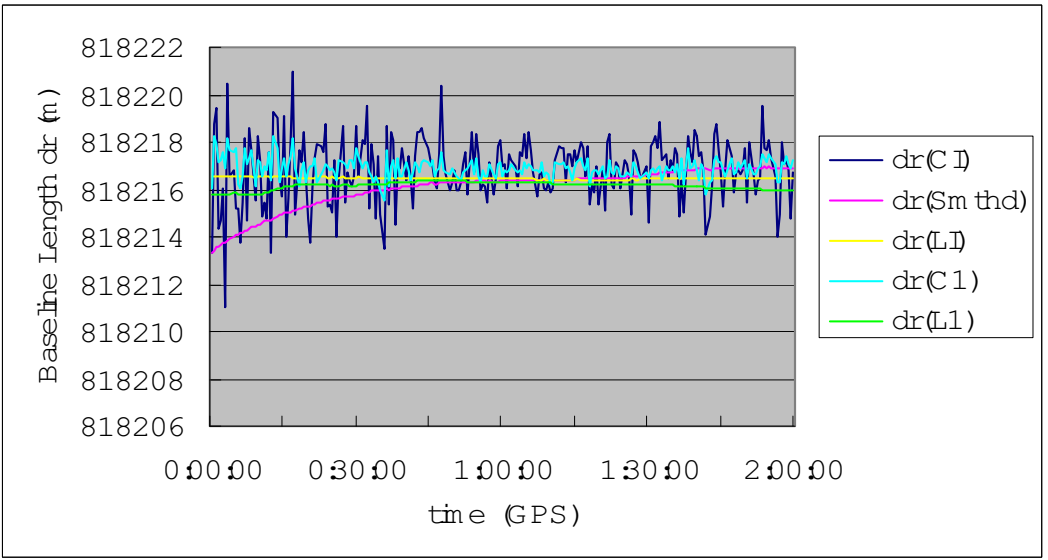

(c) $\mathrm{M}=120$

Fig. 6. Baseline lengths $d r$ obtained by $P_{I \alpha \beta}^{i j}, \bar{P}_{I \alpha \beta}^{i j}, \Phi_{I \alpha \beta}^{i j}, P_{1 \alpha \beta}^{i j}$ and $\Phi_{1_{\alpha \beta}}^{i j}$. 
Table 2a. Summary of the positioning results (2006.11.06, SpprIchk).

\begin{tabular}{|c|c|c|c|c|c|}
\hline \multirow{2}{*}{ Baseline } & $\begin{array}{c}\text { Averaging } \\
\text { interval }\end{array}$ & $\begin{array}{c}\text { Mean } \\
\text { (Std. dev.) }\end{array}$ & $\begin{array}{c}\text { Mean } \\
\text { (Std. dev.) }\end{array}$ & $\begin{array}{c}\text { Mean } \\
\text { (Std. dev.) }\end{array}$ & Static \\
\cline { 3 - 6 } $\mathrm{M}=30$ & $\begin{array}{c}0: 15: 00- \\
2: 00: 00\end{array}$ & $\begin{array}{c}818216.85 \\
(0.36)\end{array}$ & $\begin{array}{c}818216.89 \\
(1.22)\end{array}$ & $\begin{array}{c}818216.76 \\
(0.27)\end{array}$ & 818216.46 \\
\hline $\mathrm{M}=60$ & $\begin{array}{c}0: 30: 00- \\
2: 00: 00\end{array}$ & $\begin{array}{c}818216.84 \\
(0.36)\end{array}$ & $\begin{array}{c}818216.93 \\
(1.12)\end{array}$ & $\begin{array}{c}818216.71 \\
(0.20)\end{array}$ & ditto \\
\hline $\mathrm{M}=120$ & $\begin{array}{c}1: 00: 00- \\
2: 00: 00\end{array}$ & $\begin{array}{c}818216.85 \\
(0.33)\end{array}$ & $\begin{array}{c}818216.91 \\
(1.04)\end{array}$ & $\begin{array}{c}818216.64 \\
(0.22)\end{array}$ & ditto \\
\hline
\end{tabular}

Table 2b. Difference of mean baseline length from the static results of LI (2006.11.06)

\begin{tabular}{|c|c|c|c|c|}
\hline Baseline & $\begin{array}{c}\text { Averaging } \\
\text { interval }\end{array}$ & $\begin{array}{c}\mathrm{C} 1 \\
(\mathrm{~m})\end{array}$ & $\begin{array}{c}\mathrm{CI} \\
(\mathrm{m})\end{array}$ & $\begin{array}{c}\text { CI-Smthd } \\
(\mathrm{m})\end{array}$ \\
\hline $\mathrm{M}=30$ & $0: 15: 00-2: 00: 00$ & +0.39 & +0.43 & +0.30 \\
\hline $\mathrm{M}=60$ & $0: 30: 00-2: 00: 00$ & +0.38 & +0.47 & +0.25 \\
\hline $\mathrm{M}=120$ & $1: 00: 00-2: 00: 00$ & +0.39 & +0.45 & +0.18 \\
\hline
\end{tabular}

Table 2c. Standard deviation (2006.11.06)

\begin{tabular}{|c|c|c|c|c|}
\hline Baseline & $\begin{array}{c}\text { Averaging } \\
\text { interval }\end{array}$ & $\begin{array}{c}\mathrm{C} 1 \\
(\mathrm{~m})\end{array}$ & $\begin{array}{c}\text { CI } \\
(\mathrm{m})\end{array}$ & $\begin{array}{c}\text { CI-Smthd } \\
(\mathrm{m})\end{array}$ \\
\hline $\mathrm{M}=30$ & $0: 15: 00-2: 00: 00$ & 0.36 & 1.22 & 0.27 \\
\hline $\mathrm{M}=60$ & $0: 30: 00-2: 00: 00$ & 0.36 & 1.12 & 0.20 \\
\hline $\mathrm{M}=120$ & $1: 00: 00-2: 00: 00$ & 0.33 & 1.04 & 0.22 \\
\hline
\end{tabular}

\subsection{RESULTS FOR AUSTRALIAN DATA}

Similar calculations were also conducted for the data obtained from the ARGN (Australian Regional GPS Network, http://www.ga.gov.au/bin/data_server/) shown in Figure 7. Two cases of data obtained on Dec. 6, 2002 and Nov. 6, 2006 for stations Darw (Darwin), Alic (Alice Springs), Cedu (Ceduna) and Tow2 (Townsville) were analyzed. The ionosphere was very active on Dec. 6, 2002 and rather quiet on Nov. 6, 2006. The epoch is 30 seconds.

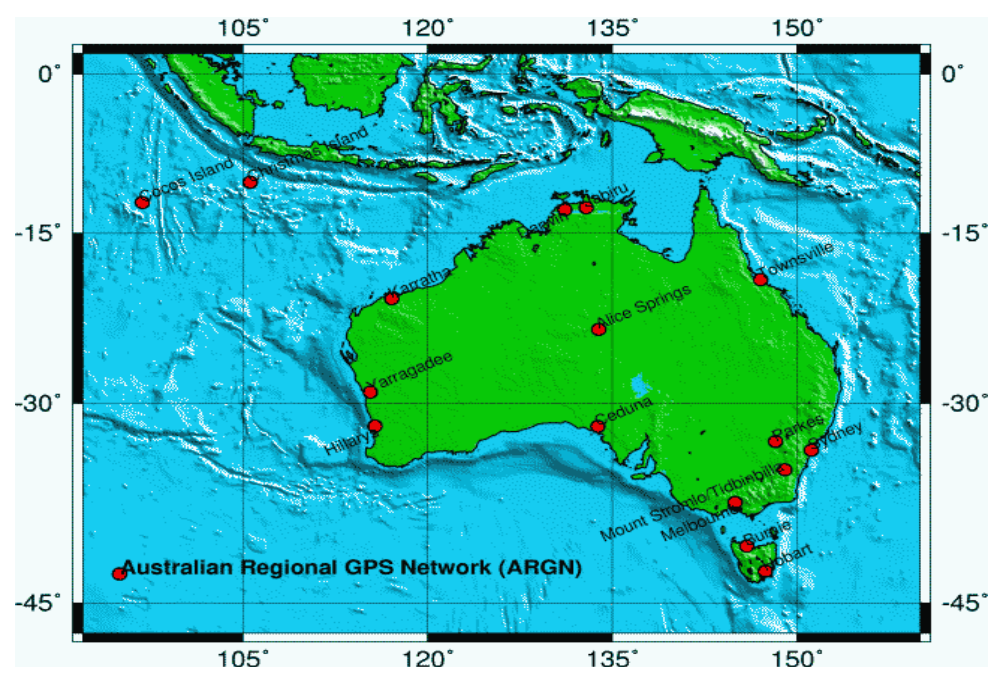

Fig. 7. Australian Regional GPS Network (ARGN) 
First, the data of 0:20:00-1:50:00 on Dec. 6, 2002 are analyzed with $M=60$. The data for satellites 2, 6, 15, 17 and 18 were used. The means and the standard deviations were obtained in an interval between 0:50:00 and 1:50:00. The results are shown in Figure 8 and Table 3. Much better results are obtained by using the CI-Smthd ranges, since the ionosphere is very active.

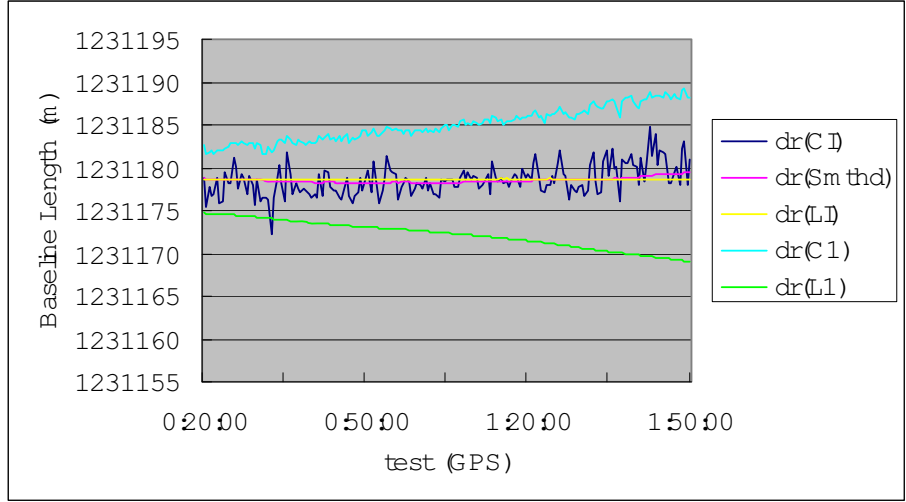

(a) Between Darw and Alic.

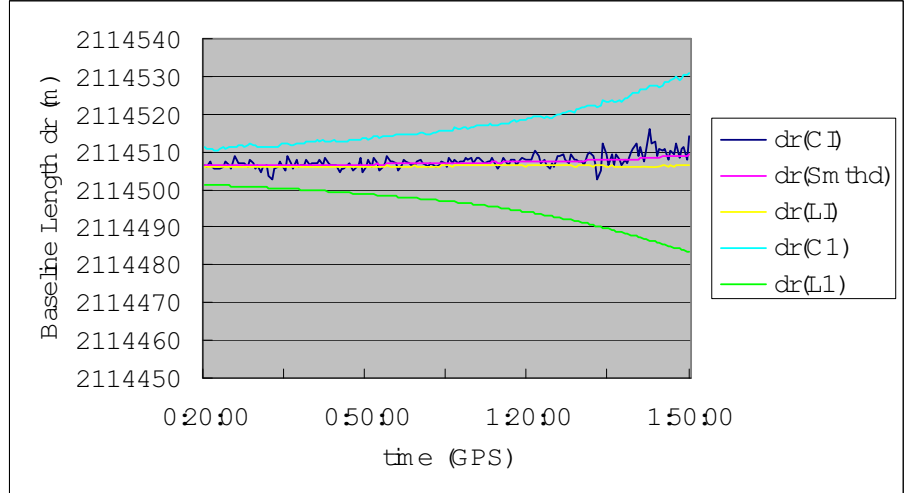

(b) Between Darw and Cedu.

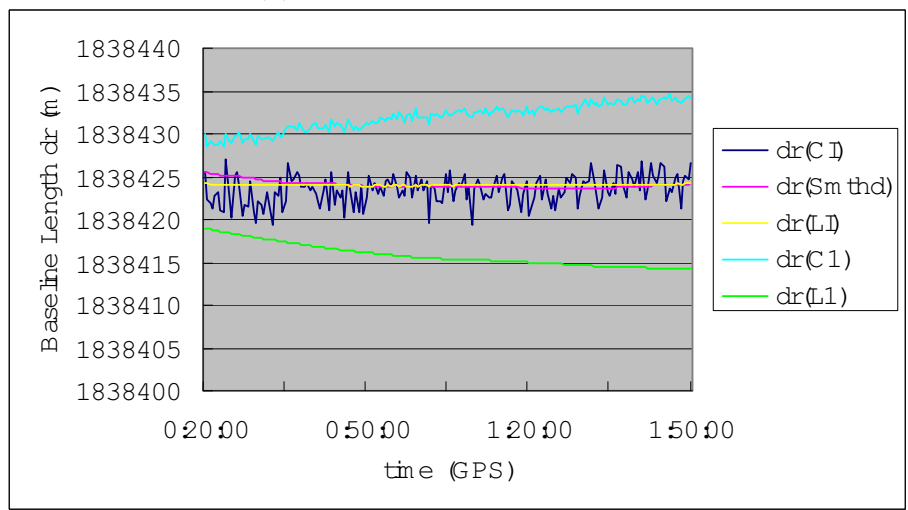

(c) Between Tow2 and Darw.

Fig. 8 (part one). Baseline lengths $d r$ between Stations obtained by $P_{I \alpha \beta}^{i j}, \bar{P}_{I \alpha \beta}^{i j}, \Phi_{I \alpha \beta}^{i j}, P_{1 \alpha \beta}^{i j}$ and $\Phi_{1 \alpha \beta}^{i j}(2002.12 .06,0: 20: 00-1: 50: 00, M=60)$. 


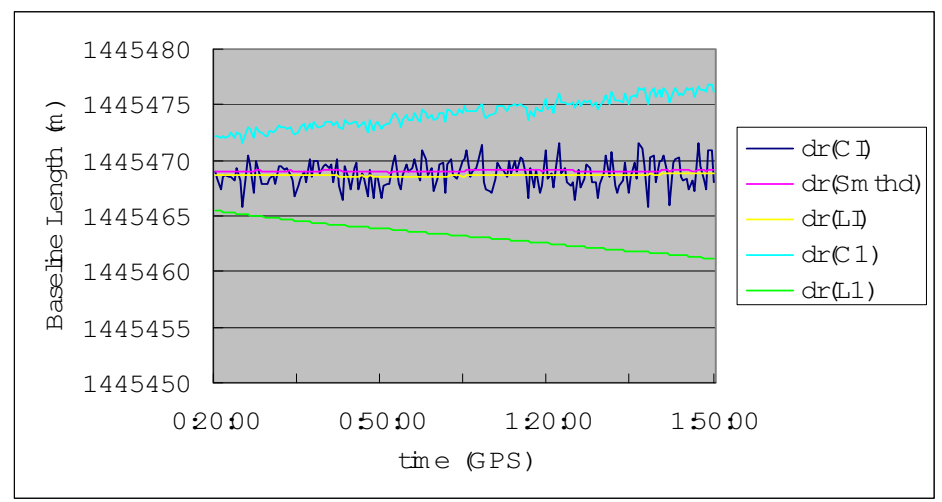

(d) Between Tow2 and Alic.

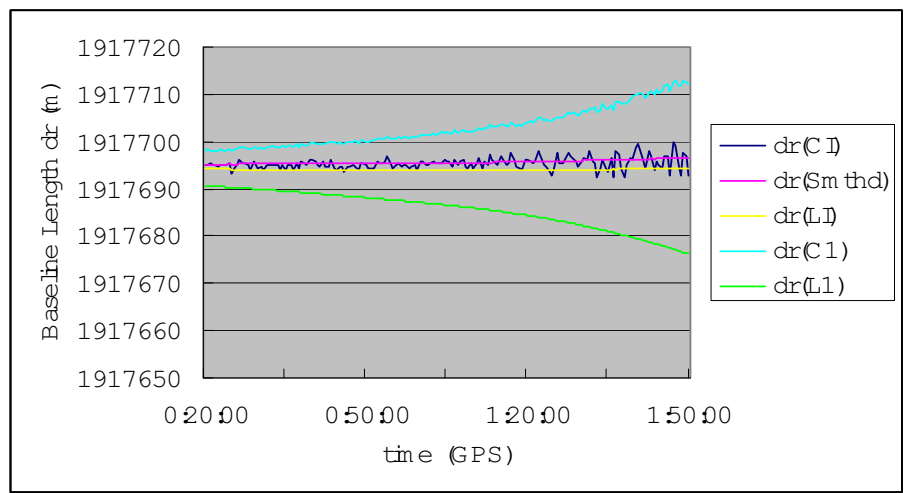

(e) Between Tow2 and Cedu.

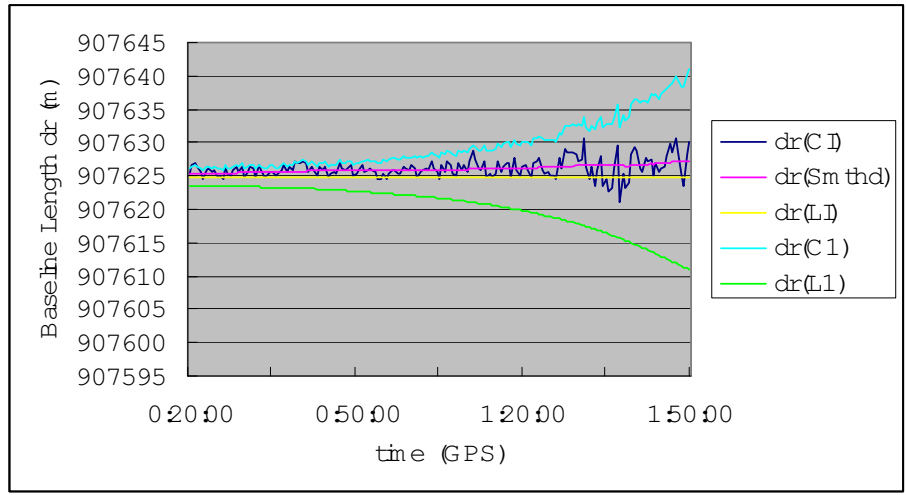

(f) Between Alic and Cedu.

Fig. 8 (part two). Baseline lengths $d r$ between Stations obtained by $P_{I \alpha \beta}^{i j}, \bar{P}_{I \alpha \beta}^{i j}, \Phi_{I \alpha \beta}^{i j}, P_{1 \alpha \beta}^{i j}$ and $\Phi_{1 \alpha \beta}^{i j}(2002.12 .06,0: 20: 00-1: 50: 00, \mathrm{M}=60)$. 
Table 3a. Summary of the positioning results (2002.12.06, 0:50:00-1:50:00).

\begin{tabular}{|l|c|c|c|c|}
\hline \multirow{2}{*}{ Baseline } & C1 & CI & CI-Smthd & LI \\
\cline { 2 - 5 } & $\begin{array}{c}\text { Mean } \\
\text { (Std. dev.) }\end{array}$ & $\begin{array}{c}\text { Mean } \\
\text { (Std. dev.) }\end{array}$ & $\begin{array}{c}\text { Mean } \\
\text { (Std. dev.) }\end{array}$ & Static \\
\hline DarwAlic & $\begin{array}{c}1231186.07 \\
(1.48)\end{array}$ & $\begin{array}{c}1231179.02 \\
(1.69)\end{array}$ & $\begin{array}{c}1231178.57 \\
(0.34)\end{array}$ & 1231178.69 \\
\hline DarwCedu & $\begin{array}{c}2114519.83 \\
(4.90)\end{array}$ & $\begin{array}{c}2114508.06 \\
(1.94)\end{array}$ & $\begin{array}{c}2114507.49 \\
(0.66)\end{array}$ & 2114506.23 \\
\hline Tow2Darw & $\begin{array}{c}1838432.87 \\
(0.87)\end{array}$ & $\begin{array}{c}1838423.74 \\
(1.50)\end{array}$ & $\begin{array}{c}1838423.82 \\
(0.14)\end{array}$ & 1838424.01 \\
\hline Tow2Cedu & $\begin{array}{c}1445474.95 \\
(0.95)\end{array}$ & $\begin{array}{c}1445468.86 \\
(1.28)\end{array}$ & $\begin{array}{c}1445469.05 \\
(0.07)\end{array}$ & 1445468.67 \\
\hline AlicCedu & $\begin{array}{c}907631.10) \\
(3.68)\end{array}$ & $\begin{array}{c}1917695.58 \\
(1.26)\end{array}$ & $\begin{array}{c}1917695.79 \\
(0.38)\end{array}$ & 1917694.06 \\
\hline
\end{tabular}

Table 3b. Difference of mean baseline length from the static results of LI (2002.12.06, 0:50:00-1:50:00)

\begin{tabular}{|c|c|c|c|}
\hline Baseline & $\begin{array}{c}\mathrm{C} 1 \\
(\mathrm{~m})\end{array}$ & $\begin{array}{c}\mathrm{CI} \\
(\mathrm{m})\end{array}$ & $\begin{array}{c}\text { CI-Smthd } \\
(\mathrm{m})\end{array}$ \\
\hline DarwAlic & +7.38 & +0.33 & -0.12 \\
\hline DarwCedu & +13.6 & +1.83 & +1.26 \\
\hline Tow2Darw & +8.86 & -0.27 & -0.19 \\
\hline Tow2Alic & +6.28 & +0.19 & +0.38 \\
\hline Tow2Cedu & +10.86 & +1.52 & +1.73 \\
\hline AlicCedu & +6.42 & +1.48 & +1.52 \\
\hline
\end{tabular}

Table 3c. Standard deviation (2002.12.06, 0:50:00-1:50:00)

\begin{tabular}{|c|c|c|c|}
\hline Baseline & $\begin{array}{c}\mathrm{C} 1 \\
(\mathrm{~m})\end{array}$ & $\begin{array}{c}\text { CI } \\
(\mathrm{m})\end{array}$ & $\begin{array}{c}\text { CI-Smthd } \\
(\mathrm{m})\end{array}$ \\
\hline DarwAlic & 1.48 & 1.69 & 0.34 \\
\hline DarwCedu & 4.90 & 1.94 & 0.66 \\
\hline Tow2Darw & 0.87 & 1.50 & 0.14 \\
\hline Tow2Alic & 0.95 & 1.28 & 0.07 \\
\hline Tow2Cedu & 4.10 & 1.26 & 0.38 \\
\hline AlicCedu & 3.68 & 1.62 & 0.40 \\
\hline
\end{tabular}

Next, the data of 0:00:00-1:30:00 on Nov. 6, 2006 were analyzed with $M=60$. The data for satellites $3,7,14,15$ and 21 were used. The means and the standard deviations were obtained in an interval between 0:30:00 and 1:30:00. The results are shown in Figure 9 and Table 4. Better results were obtained by using the CI-Smthd ranges, though the ionosphere was not so active compared with that on Dec. 6, 2002. 


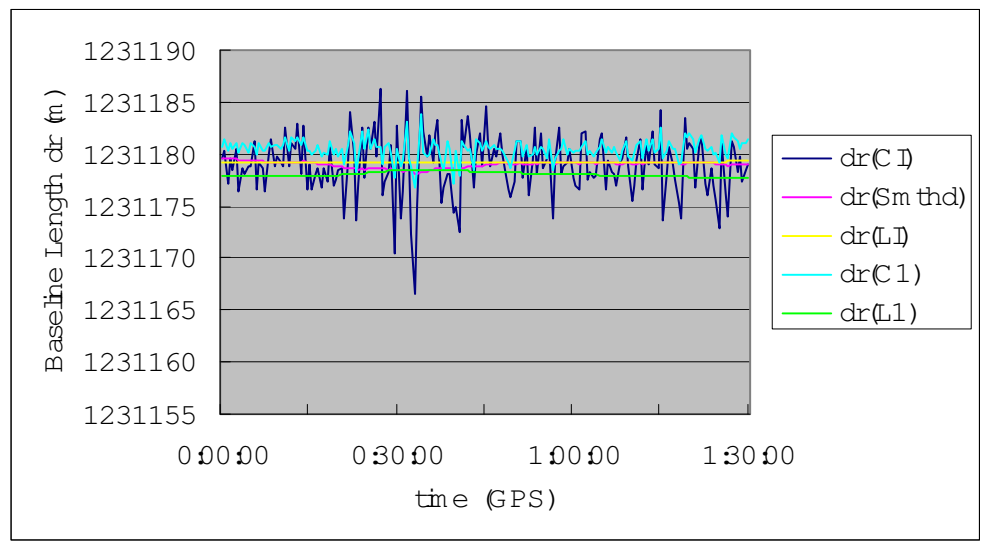

(a) Between Darw and Alic.

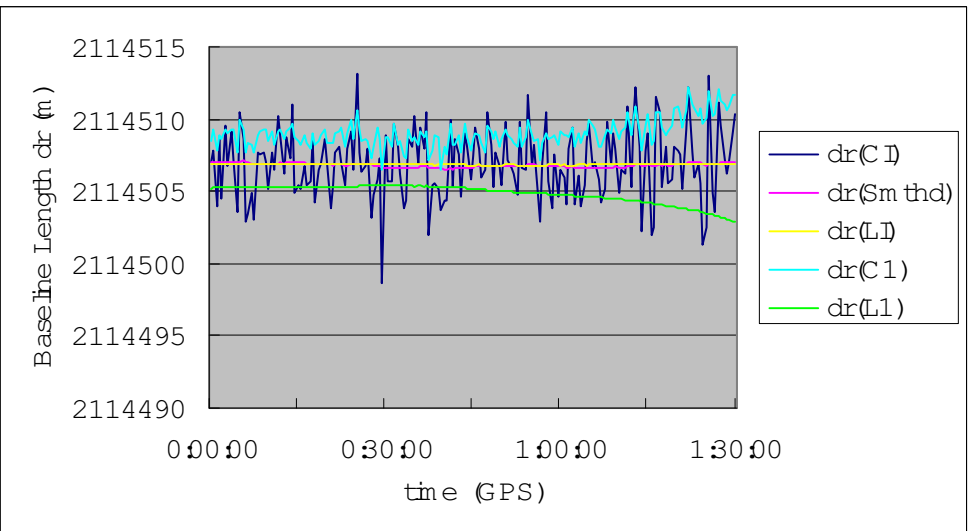

(b) Between Darw and Cedu.

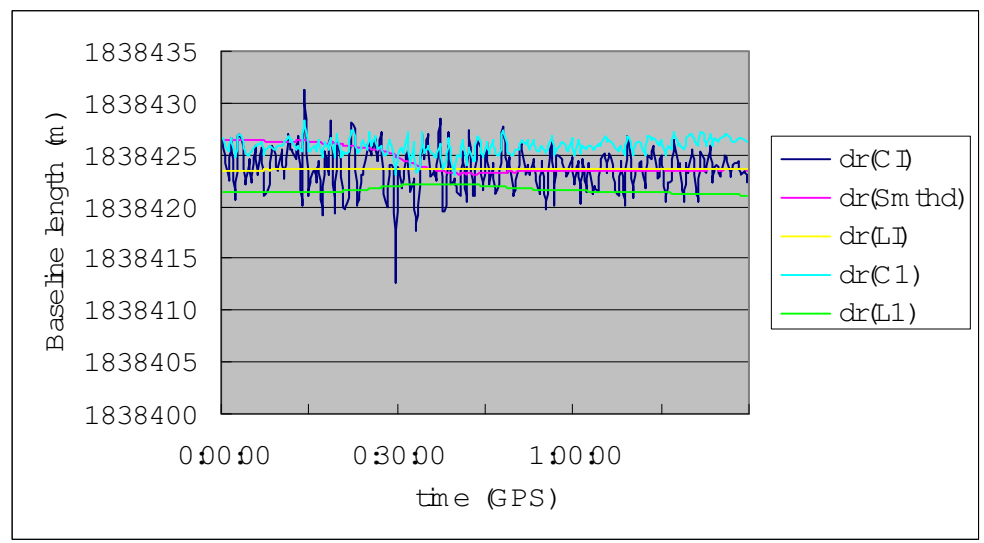

(c) Between Tow2 and Darw.

Fig. 9 (part one). Baseline lengths $d r$ between Stations obtained by $P_{I \alpha \beta}^{i j}, \bar{P}_{I \alpha \beta}^{i j}, \Phi_{I \alpha \beta}^{i j}, P_{1 \alpha \beta}^{i j}$ and $\Phi_{1 \alpha \beta}^{i j}(2006.11 .06,0: 00: 00-1: 30: 00, M=60)$. 


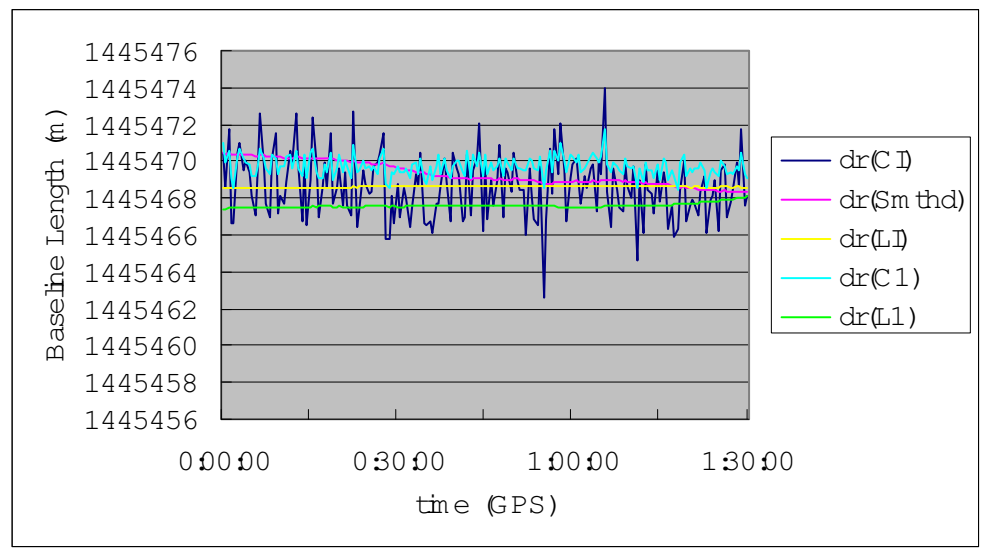

(d) Between Tow2 and Alic.

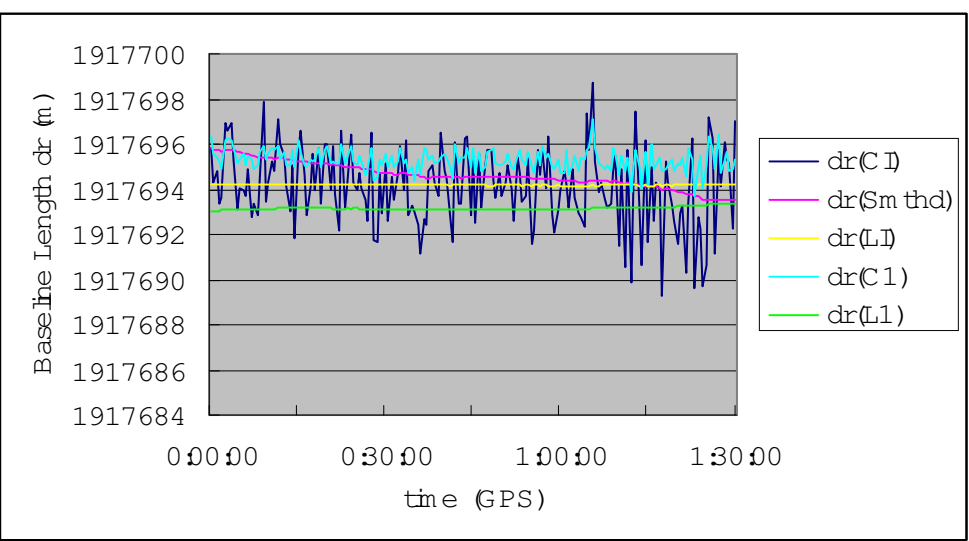

(e) Between Tow2 and Cedu.

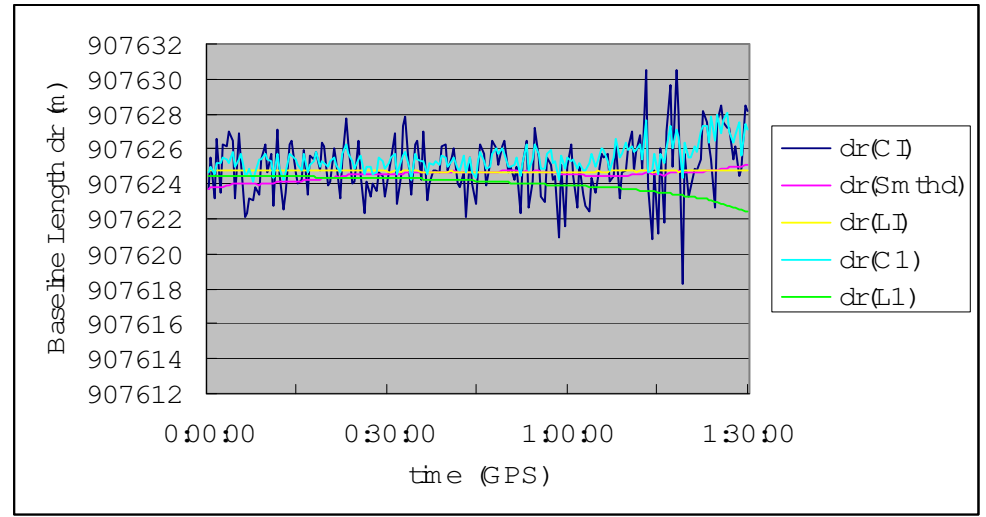

(f) Between Alic and Cedu.

Fig. 9 (part two). Baseline lengths $d r$ between Stations obtained by $P_{I \alpha \beta}^{i j}, \bar{P}_{I \alpha \beta}^{i j}, \Phi_{I \alpha \beta}^{i j}, P_{1 \alpha \beta}^{i j}$ and $\Phi_{1 \alpha \beta}^{i j}(2006.11 .06,0: 00: 00-1: 30: 00, \mathrm{M}=60)$. 
Table 4a. Summary of the positioning results (2006.11.06, 0:30:00-1:30:00).

\begin{tabular}{|c|c|c|c|c|}
\hline \multirow{2}{*}{ Baseline } & C1 & CI & CI-Smthd & LI \\
\cline { 2 - 5 } & $\begin{array}{c}\text { Mean } \\
\text { (Std. dev. })\end{array}$ & $\begin{array}{c}\text { Mean } \\
\text { (Std. dev.) }\end{array}$ & $\begin{array}{c}\text { Mean } \\
\text { (Std. dev. })\end{array}$ & Static \\
\hline DarwAlic & $\begin{array}{c}1231180.39 \\
(1.05)\end{array}$ & $\begin{array}{c}1231178.90 \\
(3.03)\end{array}$ & $\begin{array}{c}1231178.97 \\
(0.27)\end{array}$ & 1231179.18 \\
\hline DarwCedu & $\begin{array}{c}2114509.18 \\
(1.10)\end{array}$ & $\begin{array}{c}2114507.00 \\
(2.43)\end{array}$ & $\begin{array}{c}2114506.74 \\
(0.12)\end{array}$ & 2114506.86 \\
\hline Tow2Darw & $\begin{array}{c}1838425.82 \\
(0.84)\end{array}$ & $\begin{array}{c}1838423.44 \\
(1.98)\end{array}$ & $\begin{array}{c}1838423.50 \\
(0.26)\end{array}$ & 1838423.60 \\
\hline Tow2Alic & $\begin{array}{c}1445469.69 \\
(0.52)\end{array}$ & $\begin{array}{c}1445468.41 \\
(1.63)\end{array}$ & $\begin{array}{c}1445468.90 \\
(0.30)\end{array}$ & 1445468.63 \\
\hline Tow2Cedu & $\begin{array}{c}1917695.23 \\
(0.51)\end{array}$ & $\begin{array}{c}1917693.92 \\
(1.81)\end{array}$ & $\begin{array}{c}1917694.30 \\
(0.35)\end{array}$ & 1917694.22 \\
\hline AlicCedu & $\begin{array}{c}907625.67 \\
(0.79)\end{array}$ & $\begin{array}{c}907625.07 \\
(1.77)\end{array}$ & $\begin{array}{c}907624.65 \\
(0.28)\end{array}$ & 907624.74 \\
\hline
\end{tabular}

Table 4b. Difference of mean baseline length from the static results of LI (2006.11.06, 0:30:00-1:30:00)

\begin{tabular}{|c|c|c|c|}
\hline Baseline & C1 $(\mathrm{m})$ & CI $(\mathrm{m})$ & CI-Smthd(m) \\
\hline DarwAlic & +1.21 & -0.28 & -0.21 \\
\hline DarwCedu & +2.32 & +0.14 & -0.12 \\
\hline Tow2Darw & +2.22 & -0.16 & -0.10 \\
\hline Tow2Alic & +1.06 & -0.22 & +0.27 \\
\hline Tow2Cedu & +1.01 & -0.30 & +0.08 \\
\hline AlicCedu & +0.93 & +0.33 & -0.09 \\
\hline
\end{tabular}

Table 4c. Standard deviation (2006.11.06, 0:30:00-1:30:00)

\begin{tabular}{|c|c|c|c|}
\hline Baseline & C1 $(\mathrm{m})$ & CI $(\mathrm{m})$ & CI-Smthd $(\mathrm{m})$ \\
\hline DarwAlic & 1.05 & 3.03 & 0.27 \\
\hline DarwCedu & 1.10 & 2.43 & 0.12 \\
\hline Tow2Darw & 0.84 & 1.98 & 0.26 \\
\hline Tow2Alic & 0.52 & 1.63 & 0.30 \\
\hline Tow2Cedu & 0.51 & 1.81 & 0.35 \\
\hline AlicCedu & 0.79 & 1.77 & 0.28 \\
\hline
\end{tabular}

\subsection{EFFECTS OF THE TROPOSPHERE DELAYS AND THE ACCURACIES OF THE ORBITS}

The effects of the troposphere delays and the accuracies of the orbits are briefly discussed below. The static positioning results for Dec. 6, 2002 and those for Nov. 6, 2006 are compared in Table 5. Rather big differences exist for the baselines DarwAlic, DarwCedu and Tow2Darw. Although these differences do not affect the validity of the present theory, the reason is discussed below. More detailed comparisons are given in Table 6, where TO means troposphere and orbit and 1 and 0 mean the corresponding correction is on and off. As can be seen in Table 6, the effects of the troposphere delays are rather big, but those of the accuracies of the orbits are not so significant for the positioning at the decimeter level accuracy. And when the troposphere delays are neglected, the residuals of the observation equations become large. The accuracies of the estimations for troposphere delays may be responsible for the rather big differences in Table 5, and the more realistic estimations for the troposphere delays may improve the accuracies of the positioning results. 
Table 5. Comparisons of the static positioning results by LI.

\begin{tabular}{|c|c|c|c|}
\hline Baseline & $\begin{array}{c}2002.12 .06 \\
(\mathrm{~m})\end{array}$ & $\begin{array}{c}2006.11 .06 \\
(\mathrm{~m})\end{array}$ & $\begin{array}{c}\text { Difference } \\
(\mathrm{m})\end{array}$ \\
\hline DarwAlic & 1231178.69 & 1231179.18 & -0.49 \\
\hline DarwCedu & 2114506.23 & 2114506.86 & -0.63 \\
\hline Tow2Darw & 1838424.01 & 1838423.60 & 0.41 \\
\hline Tow2Alic & 1445468.67 & 1445468.63 & 0.04 \\
\hline Tow2Cedu & 1917694.06 & 1917694.22 & -0.16 \\
\hline AlicCedu & 907624.76 & 907624.74 & 0.02 \\
\hline
\end{tabular}

Table 6a. Detailed comparisons of the static positioning results between Darw and Alic results by LI.

\begin{tabular}{|c|c|c|c|c|}
\hline & TO11 $(\mathrm{m})$ & TO10 & TO01 & TO00 \\
\hline 2002.12 .06 & 1231178.69 & 1231178.60 & 1231180.43 & 1231180.35 \\
\hline 2003.12 .06 & 1231178.89 & 1231178.90 & 1231180.80 & 1231180.87 \\
\hline 2004.12 .06 & 1231179.02 & 1231178.95 & 1231180.65 & 1231180.59 \\
\hline 2005.11 .06 & 1231179.05 & 1231178.96 & 1231180.74 & 1231180.73 \\
\hline 2006.11 .06 & 1231179.18 & 1231179.22 & 1231180.42 & 1231180.54 \\
\hline
\end{tabular}

Table 6b. Detailed comparisons of the static positioning results between Darw and Alic results by LI.

\begin{tabular}{|c|c|c|c|c|c|}
\hline & $\begin{array}{c}\text { No. of } \\
\text { Satellites }\end{array}$ & TO11 $(\mathrm{m})$ & TO10-TO11 & TO01-TO11 & TO00-TO11 \\
\hline 2002.12 .06 & 5 & 1231178.69 & -0.09 & +1.74 & +1.66 \\
\hline 2003.12 .06 & 6 & 1231178.89 & +0.01 & +1.91 & +1.98 \\
\hline 2004.12 .06 & 5 & 1231179.02 & -0.07 & +1.63 & +1.57 \\
\hline 2005.11 .06 & 6 & 1231179.05 & -0.09 & +1.69 & +1.68 \\
\hline 2006.11 .06 & 5 & 1231179.18 & +0.04 & +1.24 & +1.36 \\
\hline
\end{tabular}

\subsection{UNFAVORABLE RESULTS}

Figure 10 shows the positioning results for the baseline Tow2 (Townsville) and Yar2 (Yarrangadee). The date and time of the observation are Nov. 6, 2002 and 0:00:00-2:00:00. The data for the satellites $3,7,14,15$ and 22 were used with $M=60$. Unfavorable results were obtained in this case. A comparison of the baselines obtained from the pseudo ranges $P_{1 \alpha \beta}^{i j}$ and $P_{2 \alpha \beta}^{i j}$ and the phase ranges $\Phi_{1 \alpha \beta}^{i j}$ and $\Phi_{2 \alpha \beta}^{i j}$ are given in Figure 11. As can be expected from the observation equations where the magnitudes of the ionosphere corrections are equal but the signs are opposite between the pseudo and the phase ranges, the baseline length obtained from the pseudo ranges should be symmetrical with respect to that obtained from the ionosphere free combination $\Phi_{I \alpha \beta}^{i j}$ as shown in Figure 12 corresponding to Figure 8(d). However, the results in Figure 11 violate this rule. So, we may conclude that the pseudo range $P_{2 \alpha \beta}^{i j}$ may contain significant errors. In future GNSS, $P_{2 \alpha \beta}^{i j}$ will be measured more precisely, and the situation will be improved much. 


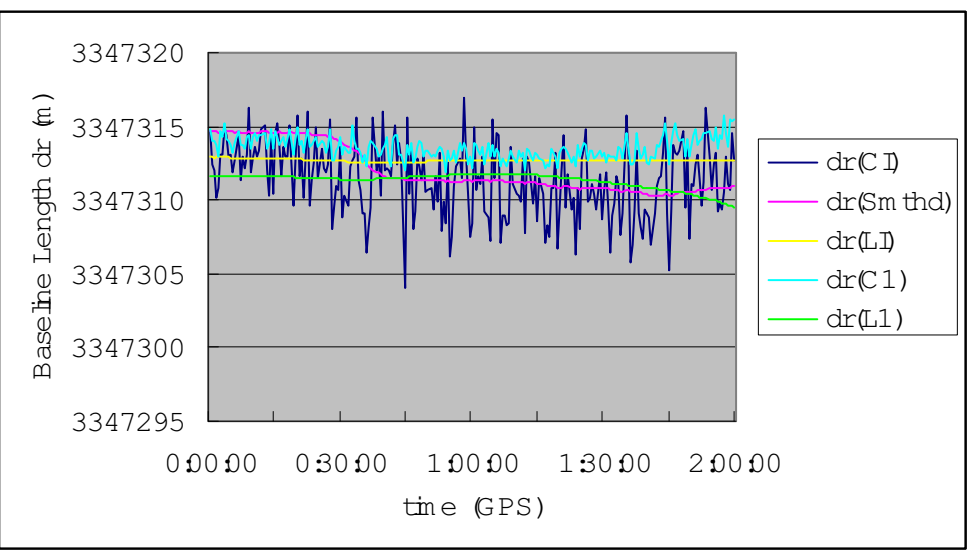

Fig. 10. Baseline lengths $d r$ between Tow 2 and Yar2 obtained by $P_{I \alpha \beta}^{i j}, \bar{P}_{I \alpha \beta}^{i j}, \Phi_{I \alpha \beta}^{i j}, P_{1 \alpha \beta}^{i j}$ and $\Phi_{1 \alpha \beta}^{i j}(2006.11 .06,0: 00: 00-2: 00: 00, M=60)$.

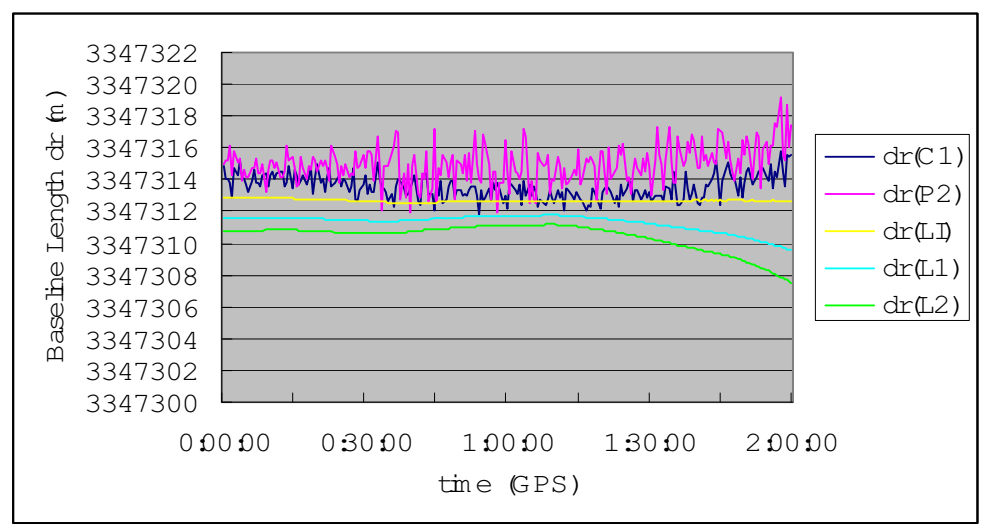

Fig. 11. Baseline lengths $d r$ between Tow 2 and Yar2 obtained by $P_{1 \alpha \beta}^{i j}, P_{2 \alpha \beta}^{i j}, \Phi_{I \alpha \beta}^{i j}, \Phi_{1 \alpha \beta}^{i j}$ and $\Phi_{2 \alpha \beta}^{i j}(2006.11 .06,0: 00: 00-2: 00: 00, M=60)$.

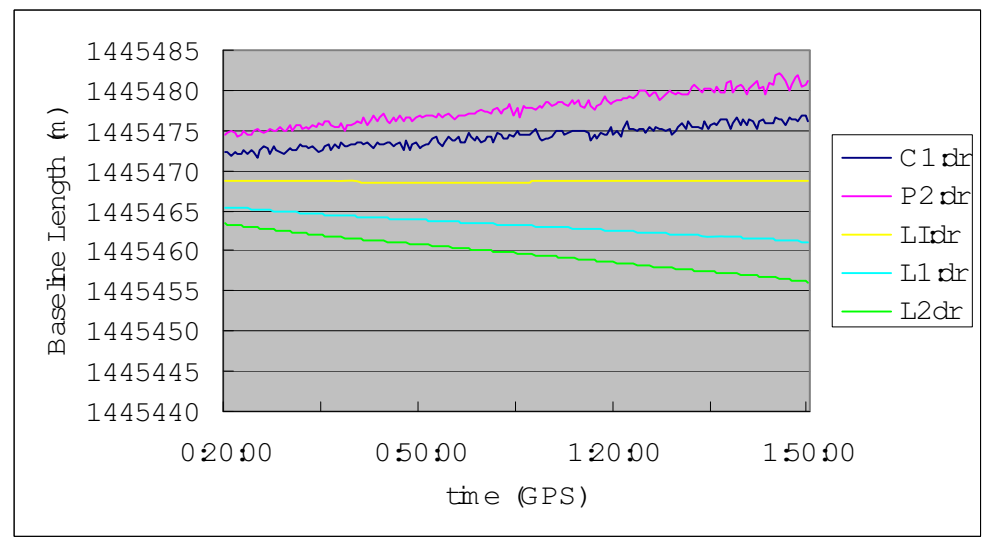

Fig. 12. Baseline lengths $d r$ between Tow 2 and Alic obtained by $P_{1 \alpha \beta}^{i j}, P_{2 \alpha \beta}^{i j}, \Phi_{I \alpha \beta}^{i j}, \Phi_{1 \alpha \beta}^{i j}$ and $\Phi_{2 \alpha \beta}^{i j}(2002.12 .06,0: 20: 00-1: 50: 00, M=60)$. 


\subsection{AN IMPROVEMENT OF THE ALGORITHM}

Because of the initial condition given by equation (4), the initial value $P_{I \alpha \beta}^{i j}(0)$ may affect the beginning part of the calculation in some cases as shown in Figure 9(e). In order to mitigate this effect, $M$ may be changed continuously, for example, as

$$
M_{1}(t)= \begin{cases}10+\frac{M-10}{M} t & \text { when } 0 \leq t \leq M \\ M & \text { when } t>M\end{cases}
$$

and the following equation is used instead of equation (3)

$$
\bar{P}_{I \alpha \beta}^{i j}(t)=\frac{1}{M_{1}(t)} P_{I \alpha \beta}^{i j}(t)+\frac{M_{1}(t)-1}{M_{1}(t)}\left[\bar{P}_{I \alpha \beta}^{i j}(t-1)+\left(\Phi_{I \alpha \beta}^{i j}(t)-\Phi_{I \alpha \beta}^{i j}(t-1)\right)\right] .
$$

The baseline length $d r$ by $\bar{P}_{I \alpha \beta}^{i j}$ or $\mathrm{dr}(\mathrm{Smth})$ shown in Figure $9(\mathrm{e})$ is recalculated according to this algorithm and the results are shown in Figure 13. An improvement in the positioning performance was obtained, which can be seen by comparing Figures 9(e) and 13.

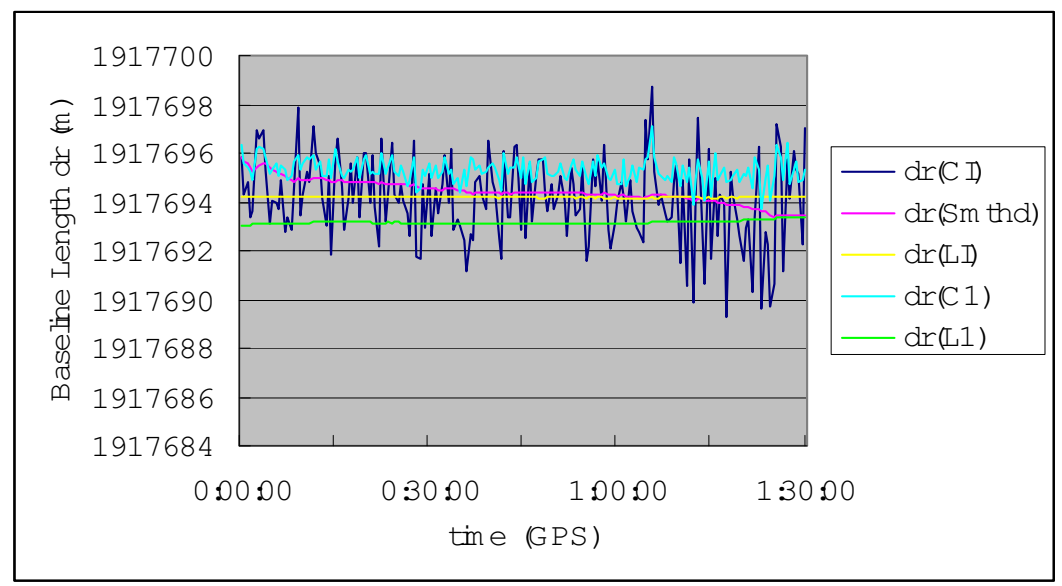

Fig. 13. Baseline lengths $d r$ between Tow2 and Cedu obtained by $P_{I \alpha \beta}^{i j}, \bar{P}_{I \alpha \beta}^{i j}, \Phi_{I \alpha \beta}^{i j}, P_{1 \alpha \beta}^{i j}$

$$
\text { and } \Phi_{1 \alpha \beta}^{i j} \quad(2006.11 .06,0: 00: 00-1: 30: 00, M=60) \text {. }
$$

\section{CONCLUSION}

A new positioning method for long baseline with dual frequency receivers is proposed. The method is based on the pseudo ranges smoothed by ionosphere free combinations of phase ranges. Since the smoothing is not affected by the ionosphere delays, the random errors in the ionosphere combinations of the pseudo ranges can be significantly eliminated as shown by the numerical examples. The smoothing may not be affected by the cycle slips, though the phase ranges are used. However, the low frequency noise components and the hardware biases in the pseudo ranges affect the positioning results. If the low frequency noise components and the hardware biases in pseudo ranges are reduced, the positioning by the smoothed pseudo ranges discussed in the present paper may be very promising. In future GNSS, the low frequency noise components and the hardware biases will be much smaller. Then, the positioning with smoothed pseudo-ranges will be very convenient and useful for many applications such as vehicle controls, since it will not be impacted by ionosphere delays (or independent of baseline 
length) and cycle slips. Furthermore, the multi-path effects may also be reduced, if the averaging interval taken is long enough.

\section{REFERENCES}

1. Misra, P. and Enge, P., (2001), Global Positioning System, Ganga-Jamuna Press, 159pp.

Received: 2007-01-16,

Reviewed: 2007-02-28, by L. Jaworski,

Accepted: 2007-04-25. 